\title{
NKT-cell defects in XLP
}

$\mathrm{X}$-linked lymphoproliferative disease (XLP) is a human immunodeficiency syndrome caused by mutations in the gene encoding the adaptor molecule SAP (signalling lymphocytic activation molecule (SLAM)-associated protein). SAPdeficient humans and mice have normal numbers of T cells, B cells and natural killer (NK) cells, but their T cells and NK cells have functional defects that lead to impaired immune responses. Now, Kim Nichols and colleagues show that SAP deficiency blocks the development of natural killer T (NKT) cells and that this seems to be a contributory factor in XLP.

SAP is known to recruit the SRCfamily kinase FYN to receptors of the SLAM family. As FYN is known to be required for the development of NKT cells, the authors speculated that NKTcell defects might contribute to XLP. Reverse-transcriptase PCR analysis showed that SAP is expressed by NKT cells from wild-type mice. When splenocytes from SAP-deficient mice were treated with the NKT-cellspecific agonist $\alpha$-galactosylceramide, they failed to produce detectable levels of interferon- $\gamma$ and interleukin- 4 . To determine whether the NKT-cell defect is quantitative or qualitative, cells from SAP-deficient mice were analysed by flow cytometry: significantly fewer NKT cells were found in SAP-deficient mice than in wild-type mice, indicating that the defect is quantitative.

The authors next asked whether the defect is intrinsic to SAP-deficient haematopoietic cells or non-haematopoietic cells. In experiments using bone-marrow chimeras, NKT cells could develop only from wild-type bone-marrow cells, so the defect is haematopoietic-cell autonomous. When 17 patients with XLP were examined, the authors observed a 97\% reduction in NKT-cell numbers in peripheral blood compared with individuals who do not have XLP. Genetic analysis of a female carrier of XLP revealed that X-chromosome inactivation was skewed in NKT cells but not in T or B cells, supporting the idea that SAP is required for NKTcell development but is dispensable for T- and B-cell development.

This study shows that SAP is crucial for the development of NKT cells in humans and mice and that lack of NKT cells might contribute to XLP. It is not clear which SLAM-family member is associated with SAP in NKT cells, and the details of the signalling pathway have yet to be worked out.

Elaine Bell

\section{(2) References and links} ORIGINAL RESEARCH PAPER Nichols, K. E. et al. Regulation of NKT cell development by SAP, the protein defective in XLP. Nature Med. 11, 340-345 (2005)

FURTHER READING Nichols, K. E. et al. Molecular and cellular pathogenesis of X-linked lymphoproliferative disease. Immunol. Rev. 203, 180-199 (2005)

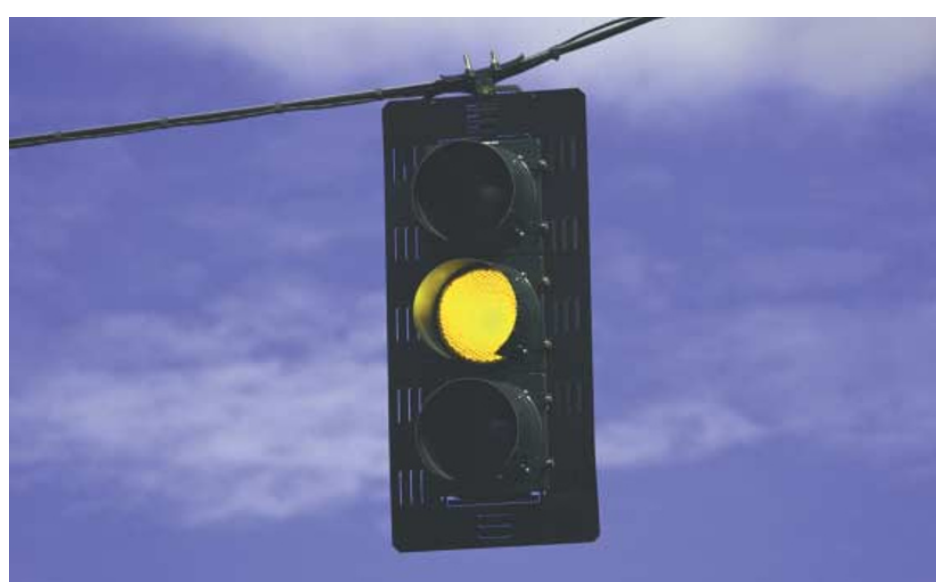

\section{IN BRIEF}

INNATE IMMUNITY

Dual role of $\alpha$-defensin- 1 in anti-HIV-1 innate immunity.

Chang, T. L. et al. J. Clin. Invest. 115, 765-773 (2005).

$\alpha$-Defensin-1 - an antimicrobial peptide that is produced by neutrophils - has been shown to inhibit HIV-1 replication. In this study, Chang et al. found that $\alpha$-defensin- 1 directly inactivates HIV-1 virions but that this inactivation is abrogated in the presence of serum or increased numbers of virus particles. Instead, in the presence of serum, $\alpha$-defensin- 1 mediates its antiviral effects on the target cells. Interestingly, protein kinase $\mathrm{C}$ (PKC) phosphorylation was reduced in $\mathrm{CD} 4^{+} \mathrm{T}$ cells treated with $\alpha$-defensin-1, and HIV-1 replication was blocked at similar stages in $\mathrm{CD}^{+} \mathrm{T}$ cells treated with either $\alpha$-defensin- 1 or an inhibitor of PKC- $\alpha$ and PKC- $\beta$. $\alpha$-Defensin- 1 , therefore, mediates its cellular effects, at least in part, through inhibition of PKC-signalling pathways.

\section{VIRAL IMMUNITY}

Inverse correlation between IL-7 receptor expression and CD8 T cell exhaustion during persistent antigen stimulation.

Lang, K. S. et al. Eur. J. Immunol. 35, 738-745 (2005).

Infection with lymphocytic choriomeningitis virus is characterized by persistence of the virus and is often associated with exhaustion of $\mathrm{CD} 8^{+} \mathrm{T}$ cells. In this study, the authors show that persistent viral antigen suppressed the expression of the interleukin-7 receptor $\alpha$-chain (IL-7R $\alpha$ ) by antigenspecific T cells. Because IL-7 is associated with the survival of memory $\mathrm{T}$ cells, prolonged downregulation of its receptor correlated with diminished $\mathrm{T}$-cell responses in chronically infected mice. By contrast, the presence of short-lived antigen resulted in only transient suppression of IL-7R $\alpha$ expression and did not cause T-cell exhaustion, indicating that antigen longevity can determine T-cell fate.

\section{T-CELL RESPONSES}

The role of herpesvirus entry mediator as a negative regulator of $\mathrm{T}$ cell-mediated responses.

Wang, Y. et al. J. Clin. Invest. 115, 711-717 (2005).

Herpesvirus entry mediator (HVEM) has previously been described as a T-cell co-stimulatory receptor, but to the surprise of these researchers, HVEM-deficient T cells, when compared with wild-type $\mathrm{T}$ cells, showed increased proliferative responses after stimulation with CD3-specific antibody or exposure to concanavalin A (conA) in vitro. Furthermore, they showed that administration of conA to HVEM-deficient mice resulted in increased morbidity and mortality, owing to the development of severe T-cell-mediated hepatitis. And compared with wild-type splenocytes, conA-treated HVEM-deficient splenocytes produced higher levels of several cytokines. Finally, HVEM-deficient mice showed increased susceptibility to experimental allergic encephalomyelitis. Together, these results indicate that HVEM can function as a negative regulator of T-cell responses. 